JOURNAL OF

APPLIED

CRYSTALLOGRAPHY

ISSN 1600-5767

\section{Handbook of Carbon Nano Materials, Volume 8, Characterization, Conducting Polymer and Sensor Applications. Edited by Francis D'Souza and Karl M. Kadish. World Scientific Press, 2016. Pp. 320. Price GBP 195.00, USD 295.00 (for the Volume 7 and 8 set). ISBN 9789814689182.}

\author{
Andrei Khlobystov*
}

School of Chemistry, University of Nottingham, University Park, Nottingham NG7 2RD, UK. *Correspondence e-mail: andrei.khlobystov@nottingham.ac.uk

If there were a prize for the most versatile chemical element in the Periodic Table, carbon would win it by a long way. The low atomic number of carbon, combined with the half-full shell of valence electrons and medium electronegativity, provides the important basis for strong covalent bonding to other carbon atoms and other elements. And this underpins the wide diversity of carbon-containing organic molecules, including the molecules of life. Indeed, all life forms on planet Earth are carbon based, and for this reason the sixth element is worthy of something close to reverence.

In many ways, recent breakthroughs in the science and technology of carbon-based nanomaterials are built on the basic chemical aspects of carbon known to chemists for a long time. However, over the past two decades the rich chemistry of carbon, combined with the limitless creativity of researchers in the field of nanoscience, has yielded proposals for a wide range of applications, including quantum computers, space elevators, new therapies for cancer, batteries, fuel cells, ultrasensitive detectors and nanoreactors. It is currently an exciting time to study carbon nanomaterials. Are carbon nanomaterials about to solve grand technological challenges and change the world around us? This is definitely the impression one gets when reading the latest research publications in this area. The sheer volume of information currently published on carbon nanomaterials can be overwhelming to experienced researchers and novices alike. The 'Handbook' makes a brave attempt to provide a definitive source of information for this highly dynamic field and presents fascinating snapshots of carbon nanomaterials research.

Volume 8, Characterization, Conducting Polymer and Sensor Applications, edited by two eminent experts in the field (F. D'Souza and K. M. Kadish), contains five chapters, each loaded with a high density of content on different aspects of nanocarbons. Because different chapters are written by different authors, there is an inevitable variation of style of presentation and narrative. For example, some chapters put specific materials in focus, such as fullerene-based polymers in Chapter 3 or iron oxide composites with graphene in Chapter 5, and build a story around the structure and properties of these materials. In contrast, Chapters 2 and 4 put key emphasis on a particular technology or application printed carbon nanomaterials for energy storage and conversion, or electrochemical sensing with carbon nanomaterials, respectively. Chapter 1 is different again, as it puts a prime emphasis on the understanding of a phenomenon (rather than a material or application) - micelle formation on a carbon nanotube surface. Despite such diversity of topics and styles, all chapters include elements of materials synthesis/processing, characterization and practical applications, albeit in different order and in different proportions, but an observant reader will see connections between the chapters within this volume. Overall, all the chapters have a good balance of fundamental science and practical application, making excellent use of the latest literature on the topic.

A certain level of didactic value present in each chapter is a welcome feature for the general understanding of the underlying physical phenomena, or specifics of material fabrication or methods of measurement, which makes the scientific material of the book more easily accessible for a general readership. Even non-expert readers with a science or engineering background should be able to get up to speed after studying the introduction 
of each chapter and to appreciate the cutting-edge research discussed in the main part. Each chapter has a good logical structure aiding the understanding, but the density of information is quite high in places, and the discussion can be tough to follow. While the illustrations and schemes are very helpful, additional tables summarizing, comparing and contrasting the key features of carbon nanomaterials or different experimental approaches would be beneficial.

All in all, this is a very timely and important book which provides a useful catalogue of ideas and an invaluable source of up-to-date information, whether for the researcher who aims to make electronic devices, sensors, or materials for energy storage or biomedical applications, or indeed for those scientists and engineers who are simply intrigued by the properties of carbon nanomaterials and would like to learn more. Despite some minor errors (such as in structural diagrams of fullerenes), the book serves as an essential guide to the synthesis, properties and applications of nanocarbons, which undoubtedly will appeal to a wide cross section of the research community. As the range of applications proposed for carbon nanomaterials becomes incredibly broad, diverse and somewhat bewildering, the Handbook makes an excellent attempt to put these into a systematic framework of knowledge and practical context. It will be fascinating to watch the development of this field over the next few years and see which of the multitude of ideas for nanoscale materials based on the sixth element will materialize as real-life technological applications. 